\title{
Age-related differences in the specificity of verbal encoding
}

\author{
ANGELA MICCO and MICHAEL E. J. MASSON \\ University of Victoria, Victoria, British Columbia, Canada
}

\begin{abstract}
A communication paradigm was used as an analogue to cued recall to separate age-related differences in encoding and retrieval. Younger and older adults (senders) generated a series of oneword clues that would enable other subjects (receivers) to generate a designated target word. Clue and target generation tasks, analogous to the encoding and retrieval components of cued recall, were conducted in the context of either a strong or a weak associate of the target. Clues generated by older senders were less effective than clues generated by younger senders in enabling receivers to generate targets, especially when clues or targets were generated in the context of a weak associate. A deficit among older receivers was also obtained, especially when a weakrather than a strong-associate context word was given to the receiver. Older adults experience difficulty with encoding and retrieval tasks that require processing of context-specific information that is not part of the generic information typically associated with a stimulus.
\end{abstract}

The memory deficits experienced by elderly adults have been characterized by Craik and Simon (1980) as a consequence of the tendency to process the general or global features of an item, rather than the context-specific information that makes an item distinctive from other events in memory. Furthermore, the lack of contextual processing is attributed to a deficit in the availability of attentional resources. General information can be encoded automatically and effortlessly, but contextual integration is effortful and requires the expenditure of processing resources that are more strictly limited in the elderly (Craik \& Byrd, 1982).

Support for the idea that the encoding processes of younger and older adults are qualitatively different has been provided by studies done with the cued recall task. In this task, memory performance can be interpreted in terms of the encoding specificity principle, by which the nature of the information encoded determines whether a retrieval cue will be effective (Tulving $\&$ Thomson, 1973). For example, Simon (1979) compared the performance of younger and older adults when memory was cued by sentence frames in which targets had been embedded at study, and when no retrieval cue was provided. Sentence cues were found to be effective only for younger adults, suggesting that integration of target words with sentence contexts during encoding was curtailed in older subjects. The effectiveness of contextual cues was also

\footnotetext{
This research was supported by Grant A7910 from the Natural Sciences and Engineering Research Council of Canada to $M$. Masson. We are grateful to David Hultsch for allowing us to make use of the pool of older adult volunteers that he has developed. We extend thanks to Ian Begg, James Chumbley, and David Mitchell for helpful comments on an earlier version of this article. Correspondence regarding this article should be addressed to Michael Masson, Department of Psychology, University of Victoria, P.O. Box 3050, Victoria, BC, Canada V8W 3P5 (e-mail address: mmasson@sol.uvic.ca).
}

found to be attenuated in a group of younger adults when the amount of study time per item was decreased. This result provides further support for the notion that limitations on available attentional resources can interfere with the encoding of contextual information.

Rabinowitz, Craik, and Ackerman (1982) further examined the proposition that older adults have a tendency to encode information at a general semantic level; they did so by conducting a replication of the original encoding specificity study reported by Tulving and Thomson (1973). In that study, during the encoding phase, each target was paired with a cue that shared either a strong or a weak semantic relationship with the target, and at the time of recall, the targets were cued either with the same cue as that used during encoding (strong-strong, weakweak) or with a cue at a different level of associative relationship to the target (strong-weak, weak-strong). In accord with the encoding specificity principle, Tulving and Thomson found that recall was more successful when the cues were the same at study and test than when the cues were changed. In the Rabinowitz et al. (1982) study, this pattern was replicated with younger adults. For the older subjects, however, a context specificity effect was found only for items encoded with a strong-associate cue (recall scores were higher in the strong-strong condition than in the strong-weak condition). No context effect was found for items encoded with a weak-associate cue (the weak-weak and the weak-strong context conditions did not differ).

Rabinowitz et al. (1982) argued that the older subjects' performance benefited in the strong-strong condition because strong cues tap general or core semantic information, and the elderly can construct general or stereotypical encodings of an event without exceeding the limits of their processing resources. They further suggested that weak-associate cues retrieve context-specific information. 
during encoding and that effortful processing was required to integrate the novel relationship between the cue and target. Older subjects do not possess the attentional resources needed to achieve an effective integration of the cue and target, and as a consequence, they did not benefit from the reinstatement of the weak-associate cue at test. This assertion was substantiated by the finding that requiring younger subjects to encode items under conditions of divided attention resulted in a failure to replicate the encoding specificity effect with weak encoding cues.

The conclusion that older adults tend to encode information at a general level has been called into question by the results of studies done with a recognition memory test and pictures rather than words (Park, Puglisi, Smith, \& Dudley, 1987; Puglisi, Park, Smith, \& Dudley, 1988). In these experiments, younger and older adults were equally sensitive to changes in context between study and test. The only circumstance in which older adults appeared to encode items in a general rather than a context-specific manner occurred when verbal materials were encoded under divided attention (Puglisi et al., 1988). On the basis of these results, Puglisi et al. (1988) suggested that the elderly are less efficient in their processing, but that the processes that they apply are not qualitatively different from those of younger adults.

Interpretation of the evidence in the debate about qualitative differences in the encoding processes of younger and older adults is complicated by the use of memory tests as a means of assessing those differences. For example, Perlmutter and Mitchell (1982) have suggested that such evaluations of differences in encoding are inadequate because they are based on circular reasoning-differences in encoding result in differences in retrieval, and when there are differences in retrieval, there must be differences in encoding. Ideally, encoding differences should be evaluated independently of retrieval.

We attempted to circumvent this difficulty in our exploration of age-related differences in encoding processes. To avoid the confounding effects of possible differences in retrieval processes, we employed the interpersonal communication paradigm (Begg, Upfold, \& Wilton, 1978; Begg \& White, 1985; Harris, Begg, \& Upfold, 1980). In this paradigm, one person, the sender, is given a target word (e.g., apple) and is required to generate a sequence of one-word clues (e.g., fruit, red, round, etc.) that would enable another person, the receiver, to produce the target. Communicability is inversely related to the number of clues needed to enable a person to produce the target. Following Begg and his colleagues, we treat the two critical components of this task-generation of cues and production of the target using the cues-as being analogous to the encoding and retrieval components of episodic memory tasks, and we assume that communicability is analogous to memorability.

Begg and White (1985) have provided justification for treating the communication paradigm as an analogue of episodic memory tasks by illustrating a number of important parallels between aspects of performance in these two domains. We review here three of these parallels. First, dimensions along which words vary, such as concreteness, have similar effects in memory and communication tasks (Begg et al., 1978). Second, variables that produce encoding specificity effects in memory tasks yield similar effects in the communication task. For instance, Begg (1978) demonstrated that for a memory task, the features that subjects use to discriminate unrelated words (e.g., beer, dog) involve category membership (beverage vs. animal), whereas the features used to discriminate categorically related words (e.g., dog, cat) are item specific (e.g., barks vs. purrs). When encoding and retrieval tasks involved the same type of discrimination, memory performance was better than it was when the two tasks demanded different types of discrimination. Similarly, in a communication task, Harris et al. (1980) required senders to produce clues that would allow receivers to discriminate a target (e.g., table) from a list of unrelated alternatives (e.g., brandy, coal, etc.) or from a list of categorically related alternatives (e.g., chair, desk, etc.). In the first case, the clues must discriminate the target at a categorical level, and in the second case, they must discriminate the target at a more specific level. The receivers were asked to choose a target item from among a set of unrelated or related distractors by using one of the two possible sets of clues. When receivers were given the set of clues that specified the target at the level appropriate to the set of distractors (i.e., category level for unrelated distractors and specific level for related distractors), they were more likely to select the target than they were if the clues specified the target at the inappropriate level.

Third, contextual factors that affect a word's interpretation during encoding affect memory and communication performance in the same way. Begg and Clark (1975) reported that concrete words were less likely to be recalled when presented in a context that biased the abstract sense of the word (e.g., set a RECORD) than when they were presented in a context that biased the concrete sense (e.g., play a RECORD). Moreover, abstract words were recalled better when presented in a context that biased a concrete sense of the word (e.g., facial FEATURE) than when presented in a context that biased the abstract sense (e.g., special FEATURE). Begg et al. (1978) reported that effectiveness of clues for concrete and for abstract targets was greater when clues were generated in a concrete context rather than an abstract context.

In general, the parallels between communication and episodic memory paradigms suggest that the informational content of verbal clues produced in the communication task is similar to that of memorial encodings. Working with this assumption, we reasoned that qualitative agerelated differences in the encoding of words could be revealed by the communication task independently of possible differences in retrieval processes. The clues generated by younger and older adults were assumed to reflect age-specific encoding processes, and these clues could be given to new subjects of either age group to simulate retrieval. In this way, the communicability of clues gener- 
ated by each age group could be assessed while holding constant the age of the subjects who used the cues to produce a target. If there are qualitative age-related differences in encoding that can be captured in the communication paradigm, they should be revealed by both age groups when those clues are used to produce a target.

The primary goal of the experiment that we report here was to test the hypothesis that there are age-related differences in encoding specificity. A variant of the communication task was patterned after the encoding specificity manipulations applied to cued recall by Rabinowitz et al. (1982). Each target word was presented in the context of a strong or a weak associate, and younger and older adults (the senders) generated a set of one-word clues that were meant to enable another person to produce the target. The receivers, who were also younger and older adults, received the clues in the presence of either the strong or the weak associate of the target. The receiver's task was to produce the target from the information provided by the associate and the clues. To simulate the cued recall conditions used by Rabinowitz et al., we manipulated the congruence between the associates provided to the sender and the receiver. Although the receivers' cues would not help to retrieve encoded information (that information was provided as the list of clues), they could be used in conjunction with the clues to help resolve the target's identity. Support for the idea that the communication paradigm is analogous to a cued recall task would be obtained if the encoding specificity effect observed in cued recall experiments were replicated in this paradigm. That is, fewer clues should be needed to produce a target when the clues had been generated in the presence of the same associate cue that was provided to the receiver (strong-strong and weak-weak conditions) than when associate cues presented during clue generation and target production were not congruent (strong-weak, weakstrong).

With respect to age-related differences in encoding processes, we hypothesized that if older adults fail to encode context-specific information, as the Rabinowitz et al. (1982) study suggested, then clues generated by older senders should be less effective than those generated by younger senders. This deficit should be particularly apparent when clues are generated in the weak-associate context, because older subjects should be less able to make use of the contextual constraint in developing their clues. When a strong-associate context is provided to senders, the age-related difference in the effectiveness of their clues should be reduced because a more generic encoding, typical of that adopted by older subjects, would be appropriate. For example, Mitchell and Perlmutter (1986) have shown that both younger and older adults are able to automatically recruit what could be considered generic knowledge related to an encoded target. The hypothesis tested here, however, is that older adults will have difficulty adhering to contextual constraints that imply a more particular interpretation of a target word. Specifically, because of limited processing resources, older adults will tend to perseverate in the recruitment of general knowledge about a target word rather than access specialized knowledge implied by a context word that is relevant to a particular interpretation of the target.

In addition, the communication paradigm allowed us to investigate age-related differences in retrieval independently of encoding differences, because the clue generation and the target production tasks were independent events. We were therefore able to test a proposition concerning retrieval differences. Specifically, if limited processing resources impair retrieval, as they are hypothesized to impair encoding (Craik \& Byrd, 1982), then younger receivers should require fewer clues to produce the target than older receivers should.

\section{METHOD}

\section{Subjects}

Thirty-eight older and 38 younger adults participated in the experiment. The sample of older adults consisted of 21 females and 17 males who ranged in age from 65 to 74 years, with a mean age of 70.2. Their mean educational level was 13.4 years. All subjects reported being in good health and lived independently in the community. They traveled to the University of Victoria campus for testing and were reimbursed $\$ 10$ for their transportation costs. The group of younger adults consisted of 32 female and 6 male undergraduates at the University of Victoria. Their mean educational level was 14.0 years. They ranged in age from 18 to 29 years, with a mean age of 21.1 , and they were provided a $\$ 5$ payment in return for their participation. Six subjects in each age group acted as senders in the experiment, and 32 subjects in each group acted as receivers. We kept the number of senders relatively small, so that each set of clues generated by a sender could be tested on more than one receiver.

\section{Design}

The experiment was constructed according to a $2 \times 2 \times 2 \times 2$ mixed factorial design. Age of receiver (younger, older) and receiver context (strong, weak associate) were between-subject factors, and age of sender (younger, older) and sender context (strong, weak associate) were within-subject factors. We manipulated the receiver age and context variables between subjects rather than the variables of age of sender or sender context because our primary interest was in the variables associated with senders and we wanted to maximize the power to detect the effects of those factors.

\section{Materials}

A set of 48 target words were selected from the Postman and Keppel (1970) word association norms, and from those used by Tulving and Thomson (1973). For each target, a strong and weak associate were also selected (e.g., glass: window, stained). For the items selected from Postman and Keppel, the strong associate was defined as the first entry on the list of the target's associates, and the weak associate was the last item on the list. For the items taken from Tulving and Thomson, the strong associate was produced by $52 \%$ of a normative sample of subjects, and the weak associate was produced by $1 \%$ of the sample (Bilodeau \& Howell, 1965; Riegel, 1965). Any target for which the strong associate was its antonym was disqualified as a candidate for selection.

Six different booklets were constructed for the clue generation task. The 48 targets were divided into eight lists of 6 targets, and each target was paired with its strong associate (e.g., glass: window). A second version of each list was constructed in which each 
target was paired with its weak associate (e.g., glass: stained). One target was selected from each of the eight lists of strong associatetarget pairings, and 1 target was selected from each of the eight lists of weak associate-target pairings. These 16 items formed one booklet. Target selection was constrained in such a way that all 16 targets in a booklet were unique. The target selection procedure was carried out six times, resulting in six booklets, each of which contained eight strong associate-target pairs and eight weak associatetarget pairs. The booklets were prepared by typing each target on a separate sheet of paper in uppercase letters. The target's associate was typed beneath it in lowercase letters, with the number 1 typed to the left of it. Six rows of underscore characters were typed directly below the associate, and the rows were numbered from 2 to 7 . Two copies of each booklet were produced, with the 16 pages of each copy arranged in an independent random order. One copy of the booklets was used for the group of younger subjects who served as senders, and the other copy was used for the older group of senders.

Each subject who served as a sender was given one booklet and was asked to produce a list of six one-word clues for each target so that another person, who would be new to the experiment, could use those clues to guess the target word's identity. Senders were told that the context word typed beneath the target was an additional clue, and that the clues that they produced should provide information that could be used in conjunction with the context word. They were instructed that when a person was tested with their clues, the context word would be given first, followed by the clues in the order in which they were produced by the sender. The objective was to enable the person to determine the target with as few clues as possible, so the senders were told that it was crucial that they give their best clue first, followed by the second best clue, and so on. Senders were instructed to work on one page of the test booklet at a time, and to commence working on the clues for the next target word only when the six clues for the target that they were currently working on had been produced.

For each of the 48 targets, the senders collectively produced one set of clues in each of four conditions defined by age of sender (younger, older) and context word given to the sender (strong, weak associate). These sets of clues were used to construct the receiver booklets given to the subjects who acted as receivers. Four basic receiver booklets were created, each containing 1 set of clues for each of the 48 targets. For each booklet, 12 of the clue sets came from each of the four conditions defined by age of sender and context word given to the sender. Within each of these groups of 12 clue sets, 2 clue sets were contributed by each of the 6 senders in the relevant age group. The assignment of clue sets to receiver booklets was counterbalanced across the four basic receiver booklets so that each of the 4 clue sets produced for a target was used once.

Two versions of each basic receiver booklet were constructed. In one version, each clue set appeared with the target's strong associate, and in the other version, each clue set appeared with the target's weak associate. Thus, in each receiver booklet, half of the clue sets were presented with the context word that was used by the sender to generate the clues, and the other half were presented with a context word that differed from the one used by the sender. The clues for a given target were typed in lowercase letters on a single page, arranged in a column according to the order in which the sender produced them, and numbered 1-6. The context word was typed in uppercase letters at the top of the page. The 48 pages of a receiver booklet were arranged in an independent random order before presentation to each receiver. Table 1 presents the four clue sets that were used for 1 of the $\mathbf{4 8}$ targets. Each of the clue sets shown in Table 1 appeared in a different basic receiver booklet.

\section{Procedure}

Receivers were tested individually and were told that on a previous day another person saw two words and produced a set of clues
Table 1

Examples of Clue Sets and the Weak-Associate Context Word for One Target Used in Receiver Booklets

Target word: CRowb

Clues from younger sender who worked with the strong associate (people):

RIOT
1. group
2. noisy
3. many
4. observing
5. cheering
6. bustling

Clues from younger sender who worked with the weak associate (riot): ROOT
1. gathering
2. people
3. push
4. shove
5. audience
6. group

Clues from older sender who worked with the strong associate (people): RIOT
1. man
2. lots
3. mob
4. flocks
5. group
6. numerous

Clues from older sender who worked with the weak associate (riot): RIOT

1. confusion

2. noisy

3. panic

4. mass

5. frenzy

6. anxious

Note-In each of the examples above, the clue set is presented with the weak associate (in uppercase letters). Each of these clue sets appeared in a different receiver booklet.

that would enable a person who was new to the experiment to generate the second word of the pair. Subjects were told that they would receive the first word of the pair and then be given a set of clues, one at a time, that would help them determine the identity of the second word in the pair (the target). Subjects were unaware that the member of the word pair that they were given (the context word) would match the context word used by the sender on only half the trials. On each trial, the page containing the context word and list of six clues was covered with a sheet of paper. The context word (e.g., WINDOW) was uncovered and read aloud by the experimenter; then, the first clue was uncovered, and the receiver attempted to generate the target word (e.g., glass). Each successive clue was uncovered, and a single candidate was orally nominated by the receiver until the target was generated or the clues were exhausted. The context word and the clues that preceded the current clue were always in the receiver's view. Half of the receivers in each age group were tested with booklets in which the strong associates of the targets were the context words, and the other half were tested with booklets in which the weak associates of the targets were the context words. Receiver context was manipulated between subjects to keep the number of trials to a reasonable level. On each trial, the number of clues required in order for the receiver to generate the target was recorded. No record was kept of the incorrect nominations. 


\section{RESULTS}

Two aspects of the data were analyzed. The first concerned the production of targets by subjects acting as receivers. The second involved an examination of selected characteristics of the clues that were generated by younger and older senders.

\section{Target Production}

Performance on the target production task was scored by counting the number of clues provided up to the point at which the receiver correctly produced the target. In cases in which a subject failed to produce a target after all six clues had been provided, a score of 7 was assigned. Failure to produce the correct target occurred on $18.5 \%$ and $26.2 \%$ of the trials involving clues generated by younger and by older senders, respectively. The mean number of clues used by receivers in their attempts to produce the target words in each condition of the experiment is shown in Table 2. Differences among these means were analyzed in an analysis of variance (ANOVA), with subjects treated as the random variable. In addition, we analyzed the data with items treated as the random variable. This analysis allowed us to demonstrate that results produced by the subjects analysis were not distorted by the influence of a few oddly behaving items (e.g., an item unfamiliar to one of the age groups), or by a sender who consistently produced inappropriate clues. This precaution was important, because there were only 6 senders in each age group. We report $F$ ratios from both analyses: $F_{1}$ refers to the subjects analysis, and $F_{2}$ refers to the items analysis. In all the analyses that we report, a Type I error rate of .05 was used (unless otherwise indicated).

In both the subjects and the items ANOVAs, age of sender, age of receiver, sender context, and receiver context were the independent variables. The analyses revealed a reliable main effect of age of sender $\left[F_{1}(1,60)=25.05\right.$, $\left.M S_{\mathrm{e}}=0.42 ; F_{2}(1,47)=12.35, M S_{\mathrm{e}}=3.83\right]$, indicating that when receivers were given clues generated by younger senders, they needed fewer clues to produce the targets than they did when they were given clues generated by older senders ( 2.95 vs. 3.36). The effect of age of sender, however, interacted with sender context $\left[F_{1}(1,60)\right.$

Table 2

Mean Number of Clues Required to Generate a Target

\begin{tabular}{|c|c|c|c|c|c|}
\hline \multirow[b]{3}{*}{ Context } & \multicolumn{4}{|c|}{ Sender Age Group and Context } & \multirow[b]{3}{*}{$M$} \\
\hline & \multicolumn{2}{|c|}{ Younger Sender } & \multicolumn{2}{|c|}{ Older Sender } & \\
\hline & Strong & Weak & Strong & Weak & \\
\hline \multicolumn{6}{|c|}{ Younger Age Group } \\
\hline $\begin{array}{l}\text { Strong } \\
\text { Weak } \\
M\end{array}$ & $\begin{array}{l}2.36 \\
3.18 \\
2.77\end{array}$ & $\begin{array}{l}2.61 \\
2.88 \\
2.75\end{array}$ & $\begin{array}{l}2.53 \\
3.72 \\
3.13\end{array}$ & $\begin{array}{l}2.99 \\
3.85 \\
3.42\end{array}$ & $\begin{array}{l}2.62 \\
3.42\end{array}$ \\
\hline \multicolumn{6}{|c|}{ Older Age Group } \\
\hline $\begin{array}{l}\text { Strong } \\
\text { Weak } \\
M\end{array}$ & $\begin{array}{l}2.80 \\
3.97 \\
3.38\end{array}$ & $\begin{array}{l}2.44 \\
3.37 \\
2.90\end{array}$ & $\begin{array}{l}2.60 \\
4.02 \\
3.31\end{array}$ & $\begin{array}{l}3.00 \\
4.14 \\
3.57\end{array}$ & $\begin{array}{l}2.71 \\
3.87\end{array}$ \\
\hline
\end{tabular}

Note-The pooled estimate of standard deviation obtained by averaging across all conditions was 0.77 .
$=7.15, M S_{\mathrm{e}}=0.61 ; F_{2}(1,47)=3.33, M S_{\mathrm{e}}=2.70 ; p<$ $.08]$ and with receiver context $\left[F_{1}(1,60)=4.86, M S_{\mathrm{e}}=\right.$ $\left.0.42 ; F_{2}(1,47)=5.02, M S_{\mathrm{e}}=1.60\right]$. These interactions are shown graphically in Figure 1.

The interaction involving age of sender and sender context, shown in the left panel of Figure 1, indicates that the effect of age of sender was nearly eliminated when the sender context was the target's strong associate. This finding is consistent with the claim that younger and older adults are both capable of encoding the general or typical semantic features of an item, but that the elderly experience difficulty when attempting to encode contextually specific information. An interesting feature apparent in the left panel of Figure 1 is that clues generated by younger senders were somewhat more effective if they were generated in conjunction with a weak rather than a strong associate of the target. This outcome reflects the fact that the encoding specificity effect (the benefit of sender and receiver using the same context word) varied across age groups, as discussed below. The interaction between age of sender and receiver context, shown in the right panel of Figure 1, indicated that the benefit of working with clues generated by younger senders was much smaller when the target was produced in the context of its strong associate. In consideration of the parallel between the communication paradigm and recall tasks, this result suggests that the encoding problems found in the elderly, and reflected in the lower effectiveness of their clues, can be overcome when information closely related to the core meaning of a target (e.g., a strong-associate context word) is available to support retrieval.

Age-related differences pertaining to retrieval processes were also evident in this study. The ANOVAs revealed a significant main effect of age of receiver $\left[F_{1}(1,60)=\right.$ $\left.6.17, M S_{e}=0.80 ; F_{2}(1,47)=8.61, M S_{e}=1.42\right]$, indicating that the younger receivers required fewer clues to produce the target than the older receivers did (3.02 vs. 3.29). In addition, presenting a strong associate as a context word for receivers was more effective than providing a weak associate $(2.67$ vs. 3.64$)\left[F_{1}(1,60)=75.97\right.$, $\left.M S_{\mathrm{e}}=0.80 ; F_{2}(1,47)=34.45, M S_{\mathrm{e}}=4.50\right]$. There also was a marginally reliable interaction between age of receiver and receiver context $\left[F_{1}(1,60)=2.90, M S_{\mathrm{e}}=\right.$ $\left.0.80, p<.10 ; F_{2}(1,47)=7.60, M S_{\mathrm{e}}=1.07\right]$. The effect of age of receiver was, according to this result, reduced when a strong rather than a weak associate was used as the receiver context (refer to the means in the last column of Table 2).

One other effect, the interaction between sender and receiver contexts, approached significance in the subjects analysis $\left[F_{1}(1,60)=3.43, M S_{\mathrm{e}}=0.55, p<.07\right]$, but was not reliable in the analysis by items. This effect, which represents the influence of encoding specificity, revealed a tendency for receivers to require fewer clues when the sender and receiver contexts were the same (3.06) rather than different (3.24). The encoding specificity effect, however, did not emerge under all conditions. The means in Table 2 are arranged so that the 


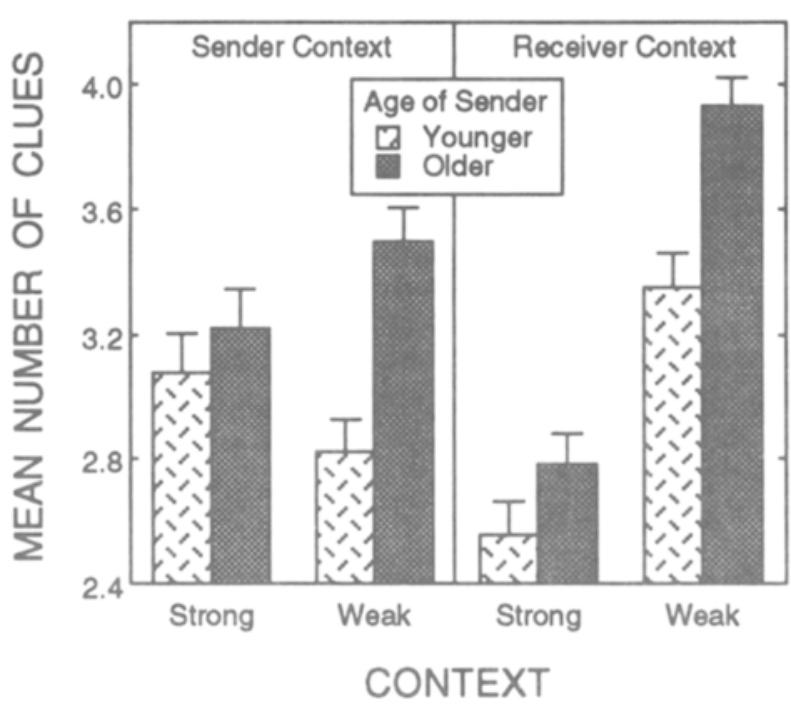

Figure 1. Mean number of clues required to produce targets as a function of age of sender and sender or receiver context. The left panel illustrates the interaction between age of sender and sender context, and the right panel shows the interaction between age of sender and receiver context. Error bars indicate one standard error of the mean.

encoding specificity effect can be seen within each combination of age of sender and age of receiver. Inspection of these conditions indicates that encoding specificity failed to occur when clues from older senders were tested with a weak associate as the receiver's context word. Both younger and older receivers produced this result, which indicates that when acting as senders, older subjects had difficulty encoding context-specific information about the target words. In addition, encoding specificity did not emerge when older receivers were tested with a strongassociate context word and younger senders' clues. If anything, there was a slight reversal of encoding specificity. We have no explanation as to why encoding specificity failed to appear in this case, but this result appears to be the reason for the slight advantage, when younger senders' clues are considered, of generating clues in the presence of a weak-associate cue (left panel of Figure 1).

Finally, two effects were reliable in the items analysis but were not significant in the subjects analysis. The interaction between age of sender and age of receiver $\left[F_{2}(1,47)\right.$ $\left.=4.31, M S_{\mathrm{e}}=0.86\right]$ and the three-way interaction of these two factors with receiver context $\left[F_{2}(1,47)=4.52\right.$, $\left.M S_{\mathrm{e}}=1.21\right]$ indicated that the advantage of younger over older receivers was strongest when receivers were tested with a weak-associate context and clues generated by younger subjects. We are reluctant to attach great importance to this outcome, because it was not anticipated and did not appear in the subjects analysis.

Under the interpretation of the communication task as an analogue of memory tasks, the obtained pattern of results suggests that older adults experience difficulties at both encoding and retrieval, especially when encoding of context-specific information is required. These deficits are attenuated, however, when the retrieval context provides support in the form of a strong-associate cue.

\section{Analysis of Clues}

We initially inspected the sets of clues generated for each target in order to determine the degree of overlap between clues generated by different senders. For each target-context pair, one set of clues was generated by 1 younger sender and another by 1 older sender. Overall, $14.1 \%$ of the clues $(16.0 \%$ for strong-associate contexts and $12.2 \%$ for weak-associate contexts) were common to the two sets of clue words for a given target-context pair. In addition, two sets of clues were generated for each target by 2 different senders in the same age group, although 1 sender was given a strong-associate context word and the other a weak associate. When the sets of clues generated by these pairs of senders were considered, it was found that, overall, $12.4 \%$ of the clues $(9.4 \%$ for younger senders and $15.3 \%$ for older senders) were common to the two sets generated for a given target. These results indicate that there was rather little overlap in the sets of clues generated in response to the same target by independently acting senders.

A second analysis of the clues was carried out to explore the basis of the differential effectiveness of clues generated by younger and older subjects. The clues were examined in an attempt to test some preliminary hypotheses regarding the characteristics of clues generated by the two age groups. The approach that we chose was to pair clue sets produced by a younger and an older sender in response to a particular target-context pair, and to have a group of raters evaluate the paired clue sets with respect to four dimensions. Each dimension referred to the relationship between a set of clues and the corresponding target.

The four dimensions for which ratings were obtained were selected to test a variety of hypotheses regarding encoding differences between younger and older adults. One dimension was intended to assess whether older adults encode distinctive contextual associations or only nonspecific information related to a target's general semantic interpretation (Hess \& Higgins, 1983). We called this dimension contextual properties and asked raters to evaluate which set of clue words best conveyed information about the relationship between the target and context words, as opposed to general information about the target's meaning. A second dimension, idiosyncratic properties, was used to determine whether older adults interpreted information in a manner that emphasized unique autobiographical experiences with a specific item. Raters were asked to indicate which set of clues conveyed information relevant to a specific, personal experience as opposed to information that would be common knowledge. The third dimension, associative properties, was used to test the hypothesis that verbal encodings of older subjects consist of fewer common associates than do verbal encodings of younger subjects (see, e.g., Perlmutter, 1979). 
Raters were to determine which set of clues in each pair contained more items that would be generated if one were asked to write down the first six words that came to mind after reading the target word. Finally, the dimension functional properties was included, because of past findings (Demming \& Pressey, 1957) that older adults are better than younger adults at answering questions concerning the "practical" features of items (e.g., the use of yellow pages in the telephone book, the type of people who might be called to do certain types of tasks, the meaning of legal terms). The raters were asked to determine which set of clues best described the task or manner in which the target item was used.

Raters. Three graduate students in psychology were recruited to rate the paired sets of clues on each of the four dimensions. Because of some difficulty in interpreting the definition of the contextual properties dimension, data on this dimension were discarded and a different set of three graduate student raters provided ratings on this dimension only. ${ }^{1}$

Materials and Procedure. For each target, the clue sets generated in the strong-associate context by the younger sender and by the older sender were paired. Similarly, the two clue sets generated in the weak-associate context, one by a younger sender and one by an older sender, were paired. The two sets of clues for a given target-context pair were typed side by side on a single sheet of paper. The target and context word appeared at the top of the page. The assignment of the younger and older senders' clues to the left and right positions was determined randomly for each pair. The resulting 96 pairs (48 targets $\times 2$ context words) of younger and older senders' clue sets were randomly arranged into a booklet.

The instruction to raters framed the clue rating task as a two-alternative forced choice task. The raters were instructed to indicate, for each of the four rating dimensions, which of the two clue sets in a pair provided the better example of the dimension. The four dimensions were defined for the raters as described earlier. The raters were unaware of the distinction between the two sets of clues that appeared with each target-context pair.

Results. For each item, we computed the proportion of raters who selected each set of clues (younger or older) for each dimension. The mean proportion of younger senders' clue sets selected on each dimension is shown in Table 3. The results for each dimension were analyzed in a separate ANOVA with age of sender and sender con-

Table 3

Mean Proportion of Raters Selecting Younger Senders' Clues

\begin{tabular}{ccc}
\hline & \multicolumn{2}{c}{ Sender Context } \\
$\begin{array}{c}\text { Rating } \\
\text { Dimension }\end{array}$ & $\begin{array}{c}\text { Strong } \\
\text { Associate }\end{array}$ & $\begin{array}{c}\text { Weak } \\
\text { Associate }\end{array}$ \\
\hline Contextual & .67 & .57 \\
Idiosyncratic & .50 & .53 \\
Associative & .64 & .70 \\
Functional & .67 & .56 \\
\hline
\end{tabular}

text as the factors. A significant age of sender effect emerged for three of the dimensions: contextual $[F(1,47)$ $\left.=12.56, M S_{\mathrm{e}}=0.23\right]$, associative $[F(1,47)=26.42$, $\left.M S_{\mathrm{e}}=0.21\right]$, and functional $\left[F(1,47)=12.35, M S_{\mathrm{e}}=\right.$ $0.20]$. For all these dimensions, the younger subjects' clues were more frequently selected as best exemplifying the properties of the rating dimension. The effect of sender context and the interaction between age of sender and context were not reliable in any of these analyses. The analysis of the idiosyncratic dimension failed to produce any reliable effects.

The results obtained for the contextual and associative dimensions were consistent with the findings of previous studies that were based on memory measures. Like the results obtained by Perlmutter (1979), the ratings suggested that the younger senders' clues consisted of more common associates than did those produced by the older senders. In addition, the younger senders' clues were rated as more effectively conveying distinctive, context-specific information than were the older senders' clues (Hess \& Higgins, 1983). The absence of an age effect on the idiosyncratic dimension is consistent with the claim of Rabinowitz et al. (1982, Experiment 1) that although the elderly tend to restrict their encoding to information of a general or prototypical nature, they do not generate idiosyncratic associations that are unlikely to be activated on a later memory test.

The finding that the younger senders' clues were more likely to be selected as best exemplifying functional properties was counter to our initial prediction that the older senders' clues would best convey this type of information. A possible reason for this discrepancy is that the Demming and Pressey (1957) paradigm consisted of answering questions about an item's functions. If they had required subjects to process the functional properties of an item in the context of an encoding task (as was involved in clue generation), age differences favoring young adults may have emerged because older adults might not be able to encode such information as effectively as younger adults.

\section{DISCUSSION}

In this study, we used a communication paradigm in which the production of target words was directly mediated by information conveyed in verbal clues. This paradigm was intended as an analogue of encoding and retrieval processes in the cued recall paradigm. In both paradigms, individuals are required to interpret information, with the end product consisting of a record of the processing event. The record is then used at a later time to reconstruct the original information. In the experiment reported here, younger and older adults generated clues for a target presented in the context of its strong or weak associate, and a different group of older and younger adults attempted to produce the target items by using these clues. Unlike cued recall tasks, this paradigm allowed us to examine age-related differences in encoding indepen- 
dently of age-related differences in retrieval by providing subjects in either age group with clues generated by either age group of senders.

Our findings were consistent with the conclusion reached by Rabinowitz et al. (1982) that older adults do not effectively integrate novel information at encoding. That is, when targets were produced using the clues generated in the weak-associate condition, the receivers in either age group who used the older senders' clues required more clues to produce the target than did the receivers who used the younger senders' clues. The need for more clues in the communication task is analogous to a memory retrieval task that is made difficult because the information (clues) that forms the encoding is of low quality. In both cases, more clues are needed to uniquely specify the target, and, as those clues are called into play, greater demands are placed on processing resources in the effort to effectively use the entire set of clues.

When targets were produced using clues generated in the strong-associate condition, however, there was very little difference in the effectiveness of younger and older senders' clues. This pattern of age effects suggests that although both the younger and the older adults were equally capable of processing the general or typical features of an item, the older adults did not incorporate context-specific information as well as the younger adults did.

Further evidence that only the younger adults effectively encoded contextual information came from the rating task. In that task, the sets of clues generated by the younger senders were more likely to be selected as best exemplifying information regarding the relationship between the context and the target word than were the clues generated by the older senders. We note that the rating data did not replicate the interaction between age of sender and sender context that was observed in the communication task. This interaction might have surfaced only in the communication task for a number of reasons. First, the rating task did not assess the magnitude of the difference between the two sets of clues in each pair. Second, in the communication task, generation of a coherent set of clues might be especially important in the context of a weak associate because the target-context relationship is highly specific (compare, for example, the strong- and weakassociate pairs doctor-nurse and doctor-witch). Given the highly specialized interpretation of the target implied by a weak associate, if the set of clues does not constitute a coherent framework within which to interpret the target, a receiver in the communication task may be misled rather badly in the effort to find the target that explains how the set of clues fits together.

The differential effectiveness of younger and older senders' clues was also modulated by the context word available to the receivers. Specifically, having a strong associate of the target available significantly reduced the effect of age of sender. This finding is important, because it is consistent with the proposal that age-related differences in recall can be reduced when retrieval support in the form of appropriate cues is provided (Craik, 1986).
Obtaining such a result in the communication paradigm reinforces the argument that the availability of retrieval support can overcome demonstrable differences in the quality of encoding.

The age-related difference that we observed among receivers is consistent with the view that older adults experience a degree of impairment in retrieval operations as well as in encoding (Craik \& Byrd, 1982; Craik \& McDowd, 1987). It has been difficult to obtain compelling evidence to support this claim, because in memory paradigms retrieval cannot be studied independently of encoding. An advantage of the communication paradigm used here was that the receiver task requirements constituted an analogue for a retrieval task in which encoding (i.e., clue generation) and target production were independent events.

\section{Comparison of Paradigms}

The value of the communication paradigm as a model for memory depends on the degree to which it captures critical features of memory encoding and retrieval. With respect to clue generation as a model of encoding, both processes involve constructing an interpretation of an item that depends on the knowledge recruited by the encoding context. When a target is processed in the context of a strong associate, the encoded interpretation emphasizes the general semantic features of the target. On the other hand, when a target is processed in the context of a weak associate, the interpretation highlights knowledge pertaining to context-specific and potentially unusual aspects of the target that were brought to the foreground by the context word. Our finding of age-related differences in the effectiveness of clues generated in the context of a weak associate indicates that older adults are less successful in the recruitment of appropriate knowledge. When more typical knowledge about an item is relevant, age differences in knowledge recruitment are not apparent (see also Mitchell \& Perlmutter, 1986).

The communication paradigm may be somewhat less faithful in its service as an analogue of memory retrieval. In the communication task, the clues developed by the sender constitute encoded information about the target, and the task of the receiver is analogous to memory retrieval in the sense that the clues (encoded information about the target) are used to reconstruct the target. In memory retrieval, however, it is also necessary to retrieve the encoded information, whereas in the communication task, that information is provided. This difference between retrieval and communication tasks suggests that retrieval context plays a somewhat different role in the two paradigms. In memory retrieval, but not in the communication task, the retrieval context would have an important influence on what encoded information was made available. We suspect that the more limited role of receiver context in the communication task is responsible for our finding of only a very weak encoding specificity effect in which target generation was slightly better when the sender and receiver contexts were the same. In contrast 
to our weak result, the cued recall task used by Rabinowitz et al. (1982) produced a powerful advantage among younger subjects for cases in which encoding and retrieval cues were matched. Older subjects in their study showed the effect as well, but only for items encoded with a strong associate.

The aspect of memory retrieval that does seem to be captured in the communication task is the interpretation of the clues and the ability to use clue information to reconstruct the target. This aspect of retrieval might be viewed as a semantic memory problem, and on the basis of earlier studies (e.g., Burke \& Harrold, 1988; Perlmutter, 1986), one might expect older subjects to perform at least as well as younger subjects. This clearly did not happen in the present study, in which younger subjects generally were more successful in generating the targets. The advantage for younger receivers, however, was apparent only when they were tested with a weak-associate context word, not when a strong associate was used. We suggest that the absence of an age-related effect when a strong-associate context word was used is due to the fact that much of the deductive process was determined by the context word, so only a few clue words were needed. When subjects were tested with weak-associate context words, however, more clue words were required, increasing the number of constraints that had to be maintained in working memory while they were deducing the target word. This account of the difficulty encountered by older subjects acting as receivers in the communication paradigm is consistent with the proposal that older adults are less able to meet the processing demands of memory retrieval tasks (Craik \& Byrd, 1982).

\section{Encoding Specificity}

Although the results of the present study provide clear support for the view that younger and older subjects differ with respect to their encoding of context-specific information, others (e.g., Park et al., 1987) who have used a recognition memory test have failed to find age differences in the encoding specificity effect like those reported by Rabinowitz et al. (1982). This outcome prompted the suggestion (Puglisi et al., 1988) that the age-related differences in context-specific encoding observed by Rabinowitz et al. (1982) may be limited to situations in which difficult retrieval tasks such as cued recall are used. The results from our use of a communication paradigm, however, suggest that the Rabinowitz et al. findings are more general than that, because encoding differences were apparent even in the absence of retrieval demands on episodic memory. We therefore do not agree that retrieval task difficulty provides a complete account of age differences in the encoding specificity effect.

A second instance in which an age difference in the encoding specificity effect fails to emerge occurs in work in which pictures have been used as stimuli (Park et al., 1987; Puglisi et al., 1988), prompting the conclusion that the age difference emerges only when verbal stimuli are used. Craik and Byrd (1982) have suggested that pictures invite deeper elaborative processing than verbal items do, and that the elderly benefit under circumstances in which the stimulus "drives" encoding processes as opposed to conditions that require effortful self-initiated processing. A similar view has been advanced by Durso and O'Sullivan (1983), who suggested that memory representations produced by pictures and words differ with regard to the "generic" information (invariant across encoding episodes) and the "specific" information (peculiar to one encoding episode) that they make available. Durso and O'Sullivan claim that pictures produce memory representations that consist primarily of information specific to the particular study episode, whereas words bias memory representations toward generic information. Viewed in this light, the failure of Puglisi et al. (1988) to observe age differences in the encoding specificity effect could have resulted from the tendency for pictures to guide encoding toward information specific to the current context. A similar argument might be put forward to account for the lack of age differences in encoding specificity when concrete word pairs, which are translated easily into mental images, have been used as stimuli (Puglisi et al., 1988)

\section{Conclusion}

The conclusion that appears to be supported by the data that we have reported and by studies done with memory paradigms is that older adults are most likely to experience difficulty when encoding context-specific information, but that the problem may not surface when stimulus materials themselves provide support for this kind of encoding. An important goal for further research is to explore the possibility that some form of guided encoding, such as generation tasks (see, e.g., Rabinowitz, 1989), may reduce or eliminate age-related deficits in encoding context-specific information, even for materials that do not strongly invite such encoding.

\section{REFERENCES}

BEGG, I. (1978). Similarity and contrast in memory for relations. Memory \& Cognition, 6, 509-517.

BegG, I., CLARK, J. M. (1975). Contextual imagery in meaning and memory. Memory \& Cognition, 3, 117-122.

BEGG, I., UPFold, D., \& WLTON, T. (1978). Imagery in verbal communication. Joumal of Mental Imagery, 2, 165-186.

BEGG, I., WHITE, P. (1985). Encoding specificity in interpersonal communication. Canadian Journal of Psychology, 39, 70-87.

Bilodeau, E. A., Howell, D. C. (1965). Free association norms (Catalog No. D210.2:F87). Washington, DC: U.S. Government Printing Office.

Burke, D. M., Harrold, R. M. (1988). Automatic and effortful semantic processes in old age: Experimental and naturalistic approaches. In L. L. Light \& D. M. Burke (Eds.), Language, memory and aging (pp. 100-116). New York: Cambridge University Press.

Craik, F. I. M. (1986). A functional account of age differences in memory. In F. Klix \& H. Hagendorf (Eds.), Human memory and cognitive capabilities: Mechanisms and performances (pp. 409-422). Amsterdam: North-Holland.

Craik, F. I. M., \& Byrd, M. (1982). Aging and cognitive deficits: The role of attentional resources. In F. I. M. Craik \& S. E. Trehub (Eds.); Aging and cognitive processes (pp. 191-211). New York: Plenum. CraIK, F. I. M., MCDowD, J. M. (1987). Age differences in recall and recognition. Joumal of Experimental Psychology: Leaming, Memory, \& Cognition, 13, 474-479. 
Craik, F. I. M., Simon, E. (1980). Age differences in memory: The role of attention and depth of processing. In L. W. Poon, J. L. Fozard, L. S. Cermak, \& L. W. Thompson (Eds.), New directions in memory and aging: Proceedings of the George A. Talland Memorial Conference (pp. 95-112). Hillsdale, NJ: Erlbaum.

Demming, J. A., \& Pressey, S. L. (1957). Tests indigenous to the adult and older years. Journal of Counselling Psychology, 4, 144-148.

Durso, F.T., \& O'Suluvan, C.S. (1983). Naming and remembering proper and common nouns and pictures. Journal of Experimental Psychology: Learning, Memory, \& Cognition, 9, 497-510.

HARRIS, G., BEGG, I., \& UPFOLD, D. (1980). On the role of the speaker's expectations in interpersonal communication. Joumal of Verbal Leaming \& Verbal Behavior, 19, 597-607.

Hess, T. M., HigGins, J. N. (1983). Context utilization in young and old adults. Journal of Gerontology, 38, 65-71.

Mrrchell, D. B. \& Perlmutter, M. (1986). Semantic activation and episodic memory: Age similarities and differences. Developmental Psychology, 22, 86-94.

Park, D. C., Puglisi, J. T., Smrth, A. D., Dudley, W. N. (1987). Cue utilization and encoding specificity in picture recognition by older adults. Joumal of Gerontology, 42, 423-425.

Perlmutter, M. (1979). Age differences in the consistency of adults' associative responses. Experimental Aging Research, 5, 549-553.

Perlmutter, M. (1986). A life-span view of memory. In P. B. Baltes, D. L. Featherman, \& R. M. Lemer (Eds.), Life-span development and behavior (Vol. 7, pp. 271-313). Hillsdale, NJ: Erlbaum.

Perlmutter, M., Mitchell, D. B. (1982). The appearance and disappearance of age differences in adult memory. In F. I. M. Craik \& S. E. Trehub (Eds.), Aging and cognitive processes (pp. 127-144). New York: Plenum.

Postman, L., KePPEL, G. (1970). Norms of word association. New York: Academic Press.
Puglisi, J. T., Park, D. C., Smith, A. D., Dudley, W. N. (1988). Age differences in encoding specificity. Joumal of Gerontology, 43, 145-150.

RABINOWITZ, J. C. (1989). Judgments of origin and generation effects: Comparisons between young and elderly adults. Psychology d Aging, 3, 259-268.

Rabinowitz, J. C., Craik, F. I. M., Ackerman, B. P. (1982). A processing resource account of age differences in recall. Canadian Journal of Psychology, 36, 325-344.

RIEGEL, K. F. (1965). Free associative responses to 200 stimuli of the Michigan restricted association norms. (USPHS Tech. Rep. No. 8, Grant MH07619). Ann Arbor: University of Michigan.

Simon, E. (1979). Depth and elaboration of processing in relation to age. Journal of Experimental Psychology: Human Leaming \& Memory, 5, 115-124.

Tulving, E., Thomson, D. M. (1973). Encoding specificity and retrieval processes in episodic memory. Psychological Review, 80, 352-373.

\section{NOTE}

1. The difficulty appeared to arise from the label that we initially used for this dimension, distinctive properties. For the second set of raters, we changed the label to contextual properties, which we use throughout this article. Despite the apparent inconsistency in the interpretation of this dimension among the original set of raters, the ratings obtained under these two labels produced the same pattern of results.

(Manuscript received June 25, 1991; revision accepted for publication December 2, 1991.) 